\title{
DEFORMATIONS OF STEIN STRUCTURES AND EXTENSIONS OF HOLOMORPHIC MAPPINGS
}

\author{
Franc Forstnerič AND MARKo Slapar
}

\begin{abstract}
Assume that $A$ is a closed complex subvariety of a Stein manifold $X$ and that $f: X \rightarrow Y$ is a continuous map to a complex manifold $Y$ such that the restriction $\left.f\right|_{A}: A \rightarrow Y$ is holomorphic on $A$. After a homotopic deformation of the Stein structure outside a neighborhood of $A$ in $X$ we find a holomorphic map $\widetilde{f}: X \rightarrow Y$ which agrees with $f$ on $A$ and which is homotopic to $f$ relative to $A$. When $\operatorname{dim}_{\mathbb{C}} X=2$ we must also change the $\mathcal{C}^{\infty}$ structure on $X \backslash A$.
\end{abstract}

\section{Introduction}

According to a classical theorem of H. Cartan every holomorphic function on a closed complex subvariety of a Stein manifold $X$ extends to a holomorphic function on all of $X[16,19]$. This extension property fails in general for mappings $X \rightarrow Y$ to more general complex manifolds unless $Y$ enjoys a certain holomorphic flexibility property introduced in [7] and [9]. In this paper we show that Cartan's extension theorem holds for maps to an arbitrary complex manifold if we allow homotopic deformations of the complex structure (and of the underlying smooth structure when $\operatorname{dim}_{\mathbb{C}} X=2$ ) in the complement of the given subvariety in the source Stein manifold $X$. The following is a simplified version of theorem 3.1 in $\S 3$ below.

Theorem 1.1. Let $X$ be a Stein manifold with $\operatorname{dim}_{\mathbb{C}} X \neq 2$ and let $A$ be a closed complex subvariety of $X$. Given a continuous map $f: X \rightarrow Y$ to a complex manifold $Y$ such that $\left.f\right|_{A}: A \rightarrow Y$ is holomorphic, there is a homotopy $\left(J_{t}, f_{t}\right)_{t \in[0,1]}$, consisting of integrable complex structures $J_{t}$ on $X$ and of continuous maps $f_{t}: X \rightarrow Y$, satisfying the following properties:

(i) $J_{0}$ is the initial complex structure on $X, J_{t}=J_{0}$ in a neighborhood of $A$ for each $t \in[0,1]$, and $J_{1}$ is a Stein structure on $X$;

(ii) $f_{0}=f,\left.f_{t}\right|_{A}=\left.f\right|_{A}$ for every $t \in[0,1]$, and $f_{1}: X \rightarrow Y$ is $J_{1}$-holomorphic.

This result is a relative version (with interpolation) of theorem 1.1 in [10] to the effect that every continuous map $f: X \rightarrow Y$ from a Stein manifold $(X, J)$ of complex dimension $\neq 2$ is homotopic to a map $\widetilde{f}: X \rightarrow Y$ which is holomorphic with respect to some Stein structure $\widetilde{J}$ on $X$ that is homotopic to $J$ through a family of integrable (but not necessarily Stein) complex structures on $X$. When $\operatorname{dim}_{\mathbb{C}} X=2$ it is in general also necessary to change the smooth structure on $X$; see theorem 4.1.

Received by the editors Sept. 19, 2005.

2000 Mathematics Subject Classification. 32H02, 32Q28, 32Q30, 32Q55, 32Q60, 32T15, 57R17.

Key words and phrases. Stein manifolds, complex structures, holomorphic mappings.

Supported by grants P1-0291 and J1-6173, Republic of Slovenia. 
The first author proved in [9] that the conclusion of theorem 1.1 holds for all data $(X, A, f)$ without changing the Stein structure on $X$ if and only if the target manifold $Y$ satisfies the convex approximation property (CAP), introduced in [7], to the effect that every holomorphic map $K \rightarrow Y$ from a compact convex set $K \subset \mathbb{C}^{n}$, $n=\operatorname{dim} X+\operatorname{dim} Y$, is a uniform limit of entire maps $\mathbb{C}^{n} \rightarrow Y$. (See also [20].) Among the conditions implying CAP we mention complex homogeneity and, more generally, the existence of a finite dominating family of holomorphic sprays. For a more complete discussion of this subject see [8].

A Stein structure $J_{1}$ in theorem 1.1 can be chosen such that $\left(X, J_{1}\right)$ is biholomorphic to $\left(\Omega,\left.J\right|_{T \Omega}\right)$ for some $J$-Stein domain $\Omega \subset X$ which contains $A$ and is diffeotopic to $X$ relative to $A$. Here is the precise result; for $A=\emptyset$ this is theorem 1.2 in [10].

Theorem 1.2. Let $X$ be a Stein manifold with $\operatorname{dim}_{\mathbb{C}} X \neq 2$ and let $A$ be a closed complex subvariety of $X$. Given a continuous map $f: X \rightarrow Y$ to a complex manifold $Y$ such that $\left.f\right|_{A}: A \rightarrow Y$ is holomorphic, there exist a Stein domain $\Omega \subset X$ containing A, a holomorphic map $\widetilde{f}: \Omega \rightarrow Y$, and a diffeomorphism $h: X \rightarrow \Omega$ which is diffeotopic to $i d_{X}$ by a diffeotopy that is fixed on a neighborhood of $A$, such that the map $\tilde{f} \circ h: X \rightarrow Y$ is homotopic to $f$ relative to $A$.

Theorem 1.1 is implied by theorem 1.2 as follows. Let $h_{t}: X \rightarrow h_{t}(X) \subset X$ be a diffeotopy as in theorem 1.2, satisfying $h_{0}=i d_{X}, h_{1}=h: X \rightarrow \Omega$, and such that $h_{t}$ is the identity map in a neighborhood of $A$ for each $t \in[0,1]$. Let $J_{t}=h_{t}^{*}(J)$ denote the (unique) complex structure on $X$ satisfying $d h_{t} \circ J_{t}=J \circ d h_{t}$ on $T X$. The homotopy $\left\{J_{t}\right\}_{t \in[0,1]}$ then satisfies theorem 1.1 (i), and the map $f_{1}:=\widetilde{f} \circ h: X \rightarrow Y$ is $J_{1}$-holomorphic and satisfies theorem 1.1 (ii).

Remark 1.3. Although a Stein structure $J_{1}$ satisfying the conclusion of theorem 1.1 must in general depend on the initial map $f$, we can choose the same $J_{1}$ for all members of a compact Hausdorff family of maps; this can be seen by applying the parametric versions of the main tools as in [10]. The analogous remark applies to theorem 1.2 in which the Stein domain $\Omega \subset X$ can be chosen the same for all maps in a compact Hausdorff family.

Theorems 1.1 and 1.2 are proved in $\S 3$. The main inductive step is furnished by lemma 2.1 whose proof relies on the tools developed by Eliashberg [3] and the authors [10]. The underlying geometric construction in this paper is more intricate than the one in [10] due to the presence of a subvariety. In $\S 4$ we discuss the analogous result for maps from Stein surfaces $\left(\operatorname{dim}_{\mathbb{C}} X=2\right)$, using results of Gompf $[13,14]$.

\section{The main lemma}

An almost complex structure on an even dimensional smooth manifold $X$ is a smooth endomorphism $J \in \operatorname{End}_{\mathbb{R}}(T M)$ satisfying $J^{2}=-I d$. It gives rise to the conjugate differential $d^{c}=-J^{*} d$, defined on functions by $\left\langle d^{c} \rho, v\right\rangle=-\langle d \rho, J v\rangle$ for $v \in T X$, and the Levi form operator $d d^{c}$. The structure $J$ is integrable if every point of $X$ admits an open neighborhood $U \subset X$ and a $J$-holomorphic coordinate map of maximal rank $z=\left(z_{1}, \ldots, z_{n}\right): U \rightarrow \mathbb{C}^{n}\left(n=\frac{1}{2} \operatorname{dim}_{\mathbb{R}} X\right)$, i.e., satisfying $d z \circ J=i d z$; for a necessary and sufficient integrability condition see [23]. 
Let $\left(X, J_{X}\right)$ and $\left(Y, J_{Y}\right)$ be a pair of (almost) complex manifolds. A smooth map $f: X \rightarrow Y$ is $\left(J_{X}, J_{Y}\right)$-holomorphic if $d f \circ J_{X}=J_{Y} \circ d f$. Since the complex structure on $Y$ will be kept fixed in our proofs, we shall simply say that $f$ is $J_{X}$-holomorphic.

We assume familiarity with standard complex analytic notions such as (strong) plurisubharmonicity and (strong) pseudoconvexity $[16,19]$. Since we shall deal with several different complex structures on the same manifold, we shall often write $J$ holomorphic, $J$-Stein, $J$-plurisubharmonic, $J$-pseudoconvex, etc.

If $(X, J)$ is a Stein manifold and $K \subset L \subset X$, with $K$ compact, we shall say that $K$ is $J$-holomorphically convex in $L$ if for every $p \in L \backslash K$ there is a $J$-holomorphic function $f$ on an open set in $X$ containing $L$ such that $|f(p)|>\sup _{x \in K}|f(x)|$. When this holds with $L=X$, we say that $K$ is $\mathcal{H}(X, J)$-convex.

The following lemma is the main step in the proof of theorems 1.1 and 1.2.

Lemma 2.1. Let $\left(X, J_{X}\right)$ be a Stein manifold with $\operatorname{dim}_{\mathbb{C}} X=n \neq 2$. Let $\rho: X \rightarrow \mathbb{R}$ be a smooth strongly $J_{X}$-plurisubharmonic Morse exhaustion function, and let $c^{\prime}<c$ be regular values of $\rho$. Set $K=\left\{x \in X: \rho(x) \leq c^{\prime}\right\}, L=\{x \in X: \rho(x) \leq c\}$. Let $A$ be a closed complex subvariety of $X$.

Assume that $J$ is an almost complex structure on $X$ which is integrable in an open neighborhood $U \subset X$ of $A \cup K$ such that $J$ agrees with $J_{X}$ in a neighborhood of $A$ in $X$ and $K$ is a strongly $J$-pseudoconvex domain with $J$-Stein interior.

Let $Y$ be a complex manifold endowed with a distance function $d_{Y}$ induced by a Riemannian metric. Given a continuous map $f: X \rightarrow Y$ which is J-holomorphic in a neighborhood of $K$ and such that $\left.f\right|_{A}: A \rightarrow Y$ is $J_{X}$-holomorphic, there exists for every $\epsilon>0$ a homotopy of pairs $\left(J_{t}, f_{t}\right)(t \in[0,1])$, where $J_{t}$ is an almost complex structure on $X$ and $f_{t}: X \rightarrow Y$ is a continuous map, satisfying the following:

(i) $J_{t}$ agrees with $J_{0}=J$ in a neighborhood of $A \cup K$ for all $t \in[0,1]$,

(ii) $J_{1}$ is integrable in a neighborhood of $A \cup L$,

(iii) $L$ is a strongly $J_{1}$-pseudoconvex domain with $J_{1}$-Stein interior, and the set $K$ is $J_{1}$-holomorphically convex in $L$,

(iv) $f_{0}=f$ and $\left.f_{t}\right|_{A}=\left.f\right|_{A}$ for each $t \in[0,1]$,

(v) for every $t \in[0,1]$ the map $f_{t}$ is $J$-holomorphic in a neighborhood of $K$ and satisfies $\sup _{x \in K} d_{Y}\left(f_{t}(x), f(x)\right)<\epsilon$, and

(vi) the map $f_{1}: X \rightarrow Y$ is $J_{1}$-holomorphic in a neighborhood of $L$.

If $J$ is integrable on $X$ then all structures $J_{t}(t \in[0,1])$ can be chosen integrable.

The situation is illustrated on fig. 1: $J$ is integrable in $U \supset A \cup K$ (shown with the dashed line), $\left.f\right|_{A}$ is holomorphic with respect to the complex structure on $A$ induced by $J_{X}$, and $f$ is $J$-holomorphic in a neighborhood of $K$. The pair final $\left(J_{1}, f_{1}\right)$ enjoys the analogous properties on the larger set $A \cup L$.

Proof. We may assume that $K=\{\rho \leq-1\}$ and $L=\{\rho \leq 0\}$.

Our first goal is to replace $f$ by another map $X \rightarrow Y$ which is holomorphic in an open neighborhood of $A \cup K$ in $X$, without changing $f$ on $A$ (where it is already holomorphic) and changing it arbitrary little on $K$.

The set $K$, being strongly $J$-pseudoconvex with $J$-Stein interior, admits a basis of $J$-Stein neighborhoods. Also, since $K$ is $J_{X}$-holomorphically convex in $X$ and $J=J_{X}$ 


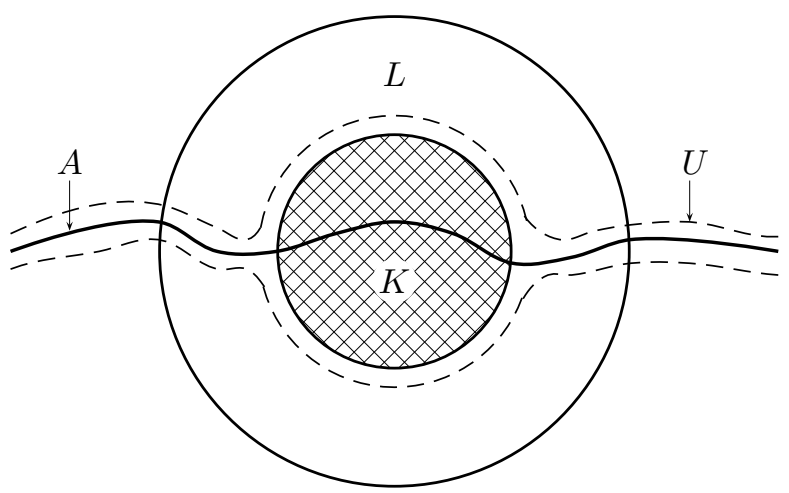

Figure 1. The main lemma.

in a neighborhood of $A$, it follows that $A \cap K$ is holomorphically convex in $A$ with respect to the complex structure induced by $J$ (or by $J_{X}$ since they agree near $A$ ). Theorem 2.1 in [9], applied to the set $A \cup K$ in the complex manifold $\left(U,\left.J\right|_{T U}\right)$, shows that $A \cup K$ admits a fundamental basis of open $J$-Stein neighborhoods $V_{j} \subset U$ such that $K$ is $J$-holomorphically convex in $V_{j}$. Replacing $U$ by such a neighborhood we shall assume that $U$ is $J$-Stein and $K$ is $\mathcal{H}(U, J)$-convex.

Theorem 3.1 in [9] now furnishes an open neighborhood $U^{\prime} \subset U$ of $A \cup K$ and a $J$-holomorphic map $f^{\prime}: U^{\prime} \rightarrow Y$ such that $\left.f^{\prime}\right|_{A}=\left.f\right|_{A}$ and $\left.f^{\prime}\right|_{K}$ is uniformly as close as desired to $\left.f\right|_{K}$. If the approximation is sufficiently close then it is possible to patch $f^{\prime}$ and $f$ outside a small open neighborhood of $A \cup K$ and thereby extend $f^{\prime}$ to all of $X$. The change from $f$ to $f^{\prime}$ is accomplished by a homotopy with the desired properties; replacing $f$ by $f^{\prime}$ and shrinking $U$ we may therefore assume $f: X \rightarrow Y$ is $J$-holomorphic in a $J$-Stein domain $U \supset A \cup K$.

Let $g_{1}, \ldots, g_{r}$ be $J_{X}$-holomorphic functions on $X$ such that

$$
A=\left\{x \in X: g_{1}(x)=0, \ldots, g_{r}(x)=0\right\} .
$$

We may assume that $\sum_{j=1}^{r}\left|g_{j}\right|^{2}<1$ on $K$. For every $\delta>0$ the function

$$
\phi_{\delta}=(\rho+1)+\delta \cdot \log \left(\sum_{j=1}^{r}\left|g_{j}\right|^{2}\right)
$$

is strongly $J_{X}$-plurisubharmonic on $X, \phi_{\delta}<0$ on $K$, and $A=\left\{\phi_{\delta}=-\infty\right\}$. A generic choice of $\delta$ insures that $\Sigma_{\delta}:=\left\{x \in L: \phi_{\delta}(x)=0\right\}$ is a smooth strongly $J_{X}$-pseudoconvex hypersurface intersecting $b L$ transversely.

We wish to smoothen the corner of the set $\left\{x \in L: \phi_{\delta}(x) \leq 0\right\}$ along $\Sigma_{\delta} \cap b L$ so that the new domain will have $J$-Stein interior and smooth strongly $J$-pseudoconvex boundary. Let $\tau_{\delta}=\operatorname{rmax}\left(\rho, \phi_{\delta}\right)$, where $r \max$ denotes a regularized maximum function [2, Lemma 5]. The function $\tau_{\delta}$ is smooth and strongly $J_{X}$-plurisubharmonic on $X$ (since $r \max$ preserves this property), it equals $\rho$ near $A$ (since $\left.\phi_{\delta}\right|_{A}=-\infty$ ), and it equals $\phi_{\delta}$ on $\left\{x \in L: \phi_{\delta} \geq 0\right\}$ (since $\rho \leq 0$ on $L$ ). The set $E_{\delta}=\left\{x \in L: \tau_{\delta}(x) \leq 0\right\}$ has smooth strongly $J_{X}$-pseudoconvex boundary which coincides with $b L$ in a neighborhood of $A \cap b L$, and it coincides with $\Sigma_{\delta}$ in $\{\rho \leq c\}$ for some $c<0$ close to 0 . 
(The set $E_{\delta}$ is shown as $D_{0}$ in fig. 2 below.) We have $K \subset \operatorname{Int} E_{\delta}$ for every $\delta>0$. As $\delta$ decreases to $0, E_{\delta}$ shrinks down to $K \cup(A \cap L)$.

We claim that for a sufficiently small $\delta>0$ the set $E_{\delta}$ has $J$-Stein interior and strongly $J$-pseudoconvex boundary $b E_{\delta}$. Since $E_{\delta}$ is contained in the $J$-Stein manifold $U$, it suffices to verify the latter property; the first one will then follow from the general theory. Recall that $J=J_{X}$ in an open set $V \supset A$. The part of $b E_{\delta}$ which belongs to $V$ is strongly $J$-pseudoconvex since $J=J_{X}$ in $V$. The remaining part $b E_{\delta} \cap(L \backslash V)$ converges to $b K \backslash V$ in the $\mathcal{C}^{\infty}$ topology as $\delta$ decreases to 0 as is seen from the definition of $\phi_{\delta}$. Since $b K$ is assumed strongly $J$-pseudoconvex, $b E_{\delta} \backslash V$ is also such provided that $\delta>0$ is chosen sufficiently small. This establishes the claim.

We now fix a $\delta>0$ satisfying the above requirements and set $\tau=\tau_{\delta}, E=E_{\delta}$. We proceed as in the proof of Lemma 6.9 in [5]. For $t \in[0,1]$ we set

$$
\rho_{t}=(1-t) \tau+t \rho, \quad D_{t}=\left\{x \in X: \rho_{t}(x) \leq 0\right\} .
$$

The function $\rho_{t}$, being a convex combination of two strongly $J_{X}$-plurisubharmonic functions $\rho_{0}=\tau$ and $\rho_{1}=\rho$, is itself strongly $J_{X}$-plurisubharmonic. The sets $D_{t}$ are strongly $J_{X}$-pseudoconvex with smooth boundaries, except at points in $b D_{t}$ where $d \rho_{t}=0$. We have $E=D_{0} \subset D_{t} \subset D_{1}=L$ for every $t \in[0,1]$; as $t$ increases from 0 to 1 , the domains $D_{t}$ monotonically increase from $D_{0}$ to $D_{1}=L$ (fig. 2).

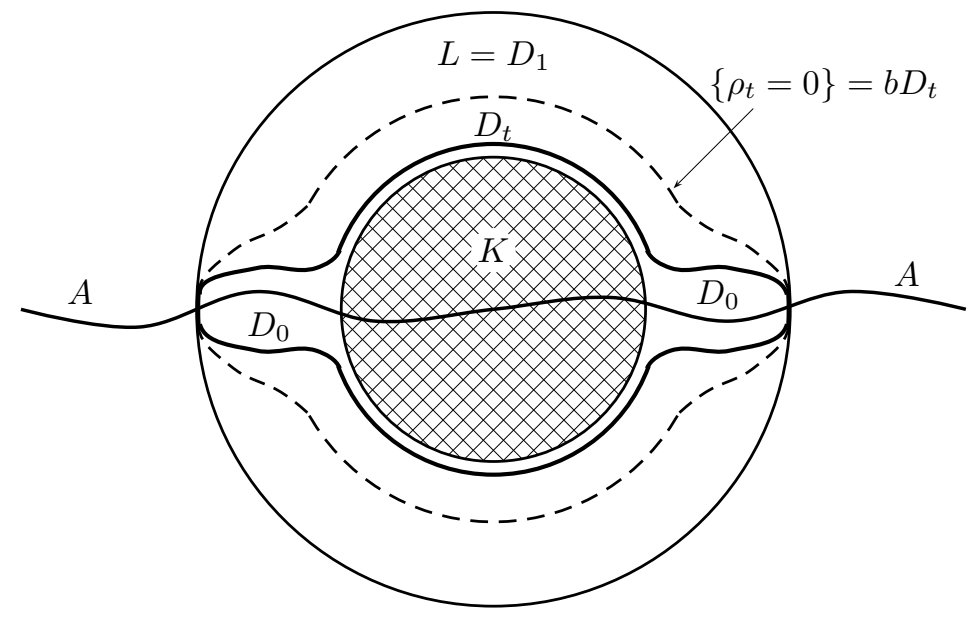

Figure 2. The sets $D_{t}=\left\{\rho_{t} \leq 0\right\}$.

Our goal is to show that the domain $L=D_{1}$ can be obtained (up to a diffeomorphism) from the domain $D_{0}$ by attaching handles of indices $\leq n$. To this end we investigate the singular points of the hypersurfaces $b D_{t}=\left\{\rho_{t}=0\right\}$ for $t \in[0,1]$. By the construction, all these boundaries coincide on $\{\rho=0, \tau=0\}=b L \cap b D_{0}$, and this set is a relative neighborhood of $A \cap b L$ in $b L$. Since the boundaries $b D_{0}=\{\tau=0\}$ and $b L=\{\rho=0\}$ are smooth and they intersect transversely along $b L \cap b D_{0}$, we see that all nonsmooth points of $b D_{t}$ are contained in the open set $\Omega=\{\rho<0, \tau>0\}=\operatorname{Int} L \backslash D_{0}$. 
The defining equation of $D_{t} \cap \Omega$ is $\tau \leq t(\tau-\rho)$; dividing by $\tau-\rho>0$ we get

$$
D_{t} \cap \Omega=\left\{x \in \Omega: \sigma(x):=\frac{\tau(x)}{\tau(x)-\rho(x)} \leq t\right\}, \quad t \in[0,1] .
$$

The critical point equation $d \sigma=0$ is equivalent to

$$
(\tau-\rho) d \tau-\tau(d \tau-d \rho)=\tau d \rho-\rho d \tau=0 .
$$

Generic choices of $\rho$ and $\tau$ insure that there are at most finitely many solutions $p_{1}, \ldots, p_{m} \in \Omega$, all nondegenerate (Morse) and belonging to pairwise distinct level sets of $\sigma$, and there are no solution on $b \Omega$. A calculation gives the following relationship between the $J_{X}$-Levi forms of these functions at a critical point $p_{j}$ of $\sigma$ :

$$
\left(\tau\left(p_{j}\right)-\rho\left(p_{j}\right)\right)^{2} \mathcal{L}_{\sigma}\left(p_{j}\right)=\tau\left(p_{j}\right) \mathcal{L}_{\rho}\left(p_{j}\right)-\rho\left(p_{j}\right) \mathcal{L}_{\tau}\left(p_{j}\right) .
$$

(In local holomorphic coordinates $z=\left(z_{1}, \ldots, z_{n}\right)$ at $p$, and with $w \in \mathbb{C}^{n}$, we have $\mathcal{L}_{\sigma}(p) \cdot w=\sum_{j, k=1}^{n} \frac{\partial \sigma}{\partial z_{j} \partial \bar{z}_{k}}(p) w_{j} \bar{w}_{k}$, and $\mathcal{L}_{\sigma}(p)>0$ means that this expression is positive for every $w \neq 0$.) Since $\tau\left(p_{j}\right)>0,-\rho\left(p_{j}\right)>0$ and the functions $\tau$ and $\sigma$ are strongly $J_{X}$-plurisubharmonic, we obtain $\mathcal{L}_{\sigma}\left(p_{j}\right)>0$. It follows that the Morse index of $\sigma$ at $p_{j}$ is $\leq n=\operatorname{dim}_{\mathbb{C}} X$. (If not, the $\mathbb{R}$-linear subspace $\Lambda$ of $T_{p_{j}} X$, corresponding to all the negative eigenvalues of the real Hessian of $\sigma$ at $p_{j}$, would have real dimension at least $n+1$ and hence $\Lambda \cap J_{X}(\Lambda)$ would contain a complex line $\lambda$; the restriction of $\mathcal{L}_{\sigma}\left(p_{j}\right)$ to $\lambda$ would therefore be negative, a contradiction.)

Choose numbers $t_{0}=0<t_{1}<t_{2}<\ldots<t_{m}=1$ which are regular values of $\left.\sigma\right|_{\Omega}$ such that $\sigma$ has exactly one critical point $p_{j} \in \Omega$ with $t_{j-1}<\rho\left(p_{j}\right)<t_{j}$ for each $j=1,2, \ldots, m$. Let $k_{j}$ denote the Morse index of $\sigma$ at $p_{j}$; thus $k_{j} \leq n$ for all $j$. By Morse theory [22] the domain $D_{t_{j}}$ is diffeomorphic to a smooth handlebody obtained by attaching a handle of index $k_{j}$ to $D_{t_{j-1}}$ and smoothing the corners (fig. 3 ).

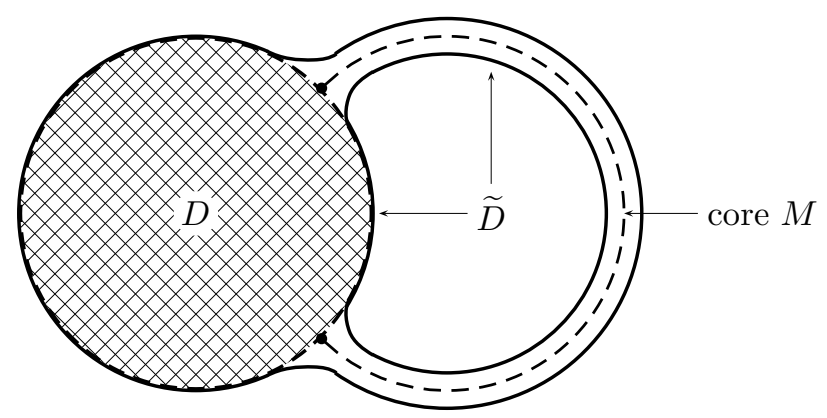

Figure 3. A handlebody $\widetilde{D}$.

Recall that a $k$-handle attached to a compact smoothly bounded domain $D \subset X$ is a diffeomorphic image of $\Delta_{k} \times \Delta_{2 n-k} \subset \mathbb{R}^{k} \times \mathbb{R}^{2 n-k}$, where $\Delta_{k}$ denotes the closed unit ball in $\mathbb{R}^{k}$. The set $b \Delta_{k} \times \Delta_{2 n-k}=S^{k-1} \times \Delta_{2 n-k}$ gets attached to $b D$, the image of $\Delta_{k} \times\{0\}^{2 n-k}$ is called the core disc (or simply the core) of the handle, and the union of $D$ with the handle, suitably smoothed at the corners, is a handlebody 
$\widetilde{D}$ shown on fig. 3. (In practice one often glues a handle to a thickening of $D$.) The Morse theory [22] tells us that every smooth manifold is obtained by successive gluing of handles, i.e., it admits a handlebody decomposition.

We are now ready to complete the proof of lemma 2.1. For consistency of notation set $W_{0}:=D_{0}$ and $J_{0}^{\prime}=J$. By what has been said, $D_{t_{1}}$ is diffeomorphic to a handlebody $W_{1} \subset D_{t_{1}}$ obtained by attaching to $W_{0}$ a handle of index $k_{1}$. Since $W_{0}$ is strongly $J$-pseudoconvex and $k_{1} \leq n \neq 2$, Eliashberg's results from [3] show that the core disc $M$ of the handle can be chosen $J$-totally real in $X$ and such that its boundary sphere $b M$ is a $J$-Legendrian (complex tangential) submanifold of $b W_{0}$. (See lemma 3.1 in [10] for details of this construction. It is here that the hypothesis $\operatorname{dim}_{\mathbb{C}} X \neq 2$ is needed; in the exceptional case $\operatorname{dim}_{\mathbb{C}} X \neq 2$ and $k_{1}=2$ it is in general impossible to find an embedded totally real core disc $M$ for the 2-handle as is shown by the gauge theory; see [3] and [13]. We shall discuss this in $\S 4$ below.)

After a small homotopic deformation of the almost complex structure $J=J_{0}^{\prime}$ supported in a neighborhood of the core disc $M$ (and away from the set $W_{0}$ ) we find a new almost complex structure $J_{1}^{\prime}$ on $X$ which is integrable near $W_{0} \cup M$ and agrees with $J_{0}^{\prime}$ near $A \cup W_{0}$, and the handlebody $W_{1}$ (a thickening of $W_{0} \cup M$ ) can be chosen such that $b W_{1}$ is smooth strongly $J_{1}^{\prime}$-pseudoconvex, Int $W_{1}$ is $J_{1}^{\prime}$-Stein, and $W_{0}$ is $J_{1}^{\prime}$-holomorphically convex in $W_{1}$. If $J$ is integrable on $X$ then the same can be accomplished without a homotopic correction of $J$ by choosing the core disc $M$ to be real analytic (see [3] and [10]).

In addition, lemma 5.1 in [10] shows that we can choose $W_{1}$ sufficiently thin around $W_{0} \cup M$ such that there exists a map $g_{1}: X \rightarrow Y$ which is $J_{1}^{\prime}$-holomorphic in a neighborhood of $W_{1}$ and satisfies the following properties:

(a) $\sup _{x \in W_{0}} d_{Y}\left(f(x), g_{1}(x)\right)<\frac{\epsilon}{m}$,

(b) $\left.g_{1}\right|_{A}=\left.f\right|_{A}$,

(c) $g_{1}$ is homotopic to $f$ by a homotopy $\left\{g_{t}\right\}_{t \in[0,1]}$ consisting of maps defined near $W_{1}$ which agree with $f$ on $A$, they are $J$-holomorphic in a neighborhood of $W_{0}$, and each of them is $\frac{\epsilon}{m}$-close to $f$ on $W_{0}$.

To obtain the interpolation conditions in (b) and (c) which are not explicitly stated by lemma 5.1 in [10], the reader should observe that the proof of that lemma relies on theorem 3.2 in [6, p. 1924] which includes interpolation on a complex subvariety.

Using the homotopy $\left\{g_{t}\right\}$ we can patch all these maps with $f$ outside a certain neighborhood of $W_{0}$ in order to get a homotopy of global maps $X \rightarrow Y$.

We now proceed to the next set $D_{t_{2}}$. By the same argument as above, $D_{t_{2}}$ is diffeomorphic to a handlebody obtained from $D_{t_{1}}$ by attaching a handle of index $k_{2}$. As $D_{t_{1}}$ is diffeomorphic to $W_{1}, D_{t_{2}}$ is also diffeomorphic to a handlebody $W_{2} \subset D_{t_{2}}$ obtained by attaching a handle of index $k_{2}$ to $W_{1}$. By repeating the above arguments we can modify $J_{1}^{\prime}$ near the core disc $M_{1}$ of the handle to a structure $J_{2}^{\prime}$ which is integrable near $W_{1} \cup M_{1}$, and we then choose $W_{2}$ to be strongly $J_{2}^{\prime}$-pseudoconvex, with $J_{2}^{\prime}$-Stein interior, and such that $W_{1}$ is $J_{2}^{\prime}$-holomorphically convex in $W_{2}$. After shrinking $W_{2}$ around $W_{1} \cup M_{1}$ we also get a map $g_{2}: X \rightarrow Y$ which is $J_{2}^{\prime}$-holomorphic in a neighborhood of $W_{2}$, it agrees with $f$ on $A$, it satisfies $\sup _{x \in W_{1}} d_{Y}\left(g_{2}(x), g_{1}(x)\right)<$ $\frac{\epsilon}{m}$, and it is homotopic to $g_{1}$ by a homotopy $\left\{g_{t}\right\}_{t \in[1,2]}$ which is fixed on $A$ such that each $g_{t}$ is $J_{1}^{\prime}$-holomorphic near $W_{1}$ and is uniformly $\frac{\epsilon}{m}$-close to $g_{1}$ on $W_{1}$. 
Continuing inductively we obtain after $m$ steps a handlebody $W_{m} \subset L$, diffeomorphic to $L$, and an almost complex structure $J_{m}^{\prime}$ on $X$ which is integrable in a neighborhood of $A \cup W_{m}$ and which agrees with $J=J_{0}^{\prime}$ in a neighborhood of $A \cup W_{0}$ (in fact, the two structures are homotopic by a homotopy that is fixed near $A \cup W_{0}$ ), such that $W_{m}$ is strongly $J_{m}^{\prime}$-pseudoconvex and its interior is $J_{m}^{\prime}$-Stein. We also obtain a map $g_{m}: X \rightarrow Y$ which is $J_{m}^{\prime}$-holomorphic in a neighborhood of $W_{m}$, it agrees with $f$ on $A$, and it satisfies $\sup _{x \in D_{0}} d_{Y}\left(f(x), g_{m}(x)\right)<\epsilon$. The construction also gives a homotopy of maps $X \rightarrow Y$ connecting $f$ to $g_{m}$ such that the homotopy is fixed on $A$, each map in the family is $J$-holomorphic in a neighborhood of $D_{0}=W_{0}$ and is uniformly $\epsilon$-close to $f$ on $D_{0}$ (and hence on $K$ ).

Our construction of the handlebodies $W_{1}, \ldots, W_{m}$ insures that there is a diffeomorphism $h: X \rightarrow X$ such that $h(L)=W_{m}$ and $h$ is diffeotopic to $i d_{X}$ by a diffeotopy that is fixed in an open neighborhood of $A \cup K$. (We may even insure that $h\left(D_{t_{j}}\right)=W_{j}$ for $j=0,1, \ldots, m$.)

Set $J_{1}=h^{*}\left(J_{m}^{\prime}\right)$ and $f_{1}=g_{m} \circ h: X \rightarrow Y$. The definition of $J_{1}$ is equivalent to $d h \circ J_{1}=J_{m}^{\prime} \circ d h$, which means that $h:\left(X, J_{1}\right) \rightarrow\left(X, J_{m}^{\prime}\right)$ is a biholomorphic map. Note that $J_{1}$ is integrable in a neighborhood of $A \cup L$ (since $J_{m}^{\prime}$ is integrable near $\left.A \cup W_{m}\right)$, and $J_{1}$ coincides with $J$ near $A$ (since $h$ is the identity near $A$ ).

If $\left\{h_{t}\right\}_{t \in[0,1]}$ is a diffeotopy on $X$ from $h_{0}=i d_{X}$ to $h_{1}=h$ which is fixed near $A \cup K$ then $J_{t}=h_{t}^{*}\left(J_{m}^{\prime}\right)$ is a homotopy of almost complex structures which is fixed in a neighborhood of $A \cup K$ and which connects $J_{0}=J$ to $J_{1}$.

If $J$ is integrable on $X$ then $J=J_{0}^{\prime}=J_{1}^{\prime}=\cdots J_{m}^{\prime}$ by the construction, and hence the structure $J_{t}$ is integrable for every $t \in[0,1]$ since conjugation by a diffeomorphism preserves integrability. This verifies properties (i) and (ii) in lemma 2.1.

The set $L=h^{-1}\left(W_{m}\right)$ is strongly $J_{1}$-pseudoconvex and its interior is $J_{1}$-Stein since $W_{m}$ enjoys these properties with respect to $J_{m}^{\prime}$. Also, $W_{j}$ is $J_{j+1}^{\prime}$-holomorphically convex in $W_{j+1}$ and $J_{j}^{\prime}=J_{j+1}^{\prime}$ near $W_{j}$ for $j=0,1, \ldots, m-1$; since $K$ is $J_{0}^{\prime}$ holomorphically convex in $U$ and hence in $W_{0}$, we see that $K$ is $J_{m}^{\prime}$-holomorphically convex in $W_{m}$. Thus $K$ is $J_{1}$-holomorphically convex in $L$ and hence (iii) holds.

The map $f_{1}=g_{m} \circ h: X \rightarrow Y$ is $J_{1}$-holomorphic near $L\left(\right.$ since $h:\left(X, J_{1}\right) \rightarrow\left(X, J_{m}^{\prime}\right)$ is biholomorphic and $g_{m}$ is $J_{m}^{\prime}$-holomorphic in a neighborhood of $W_{m}=h(L)$ ), so (vi) holds. By the construction we also have $\sup _{x \in K} d_{Y}\left(f(x), f_{1}(x)\right)<\epsilon$. A homotopy from $f=f_{0}$ to $f_{1}$ satisfying properties (iv) and $(\mathrm{v})$ is obtained by combining the individual homotopies obtained in the construction. This completes the proof.

Remark 2.2. H. Hamm proved $[17,18]$ that for every $n$-dimensional Stein space $X$ and closed complex subvariety $A \subset X$ the pair $(X, A)$ is homotopically equivalent to a relative CW complex of dimension $\leq n=\operatorname{dim}_{\mathbb{C}} X$. (The absolute version with $A=\emptyset$ is a well known theorem of Lefshetz [21], Abraham and Fraenkel [1] and Milnor [22].) In his proof Hamm used Morse theory for manifolds with boundary. The essential step is the following [18, pp. 2-5]:

Assume that $A$ is a closed complex subvariety of an $n$-dimensional Stein space $X$ such that $X \backslash A$ is regular (without singularities). Let $K \subset L$ be sublevel sets of a real analytic, strongly plurisubharmonic Morse exhaustion function on $X$. Then $(A \cap L) \cup K$ admits a thickening $D \subset L$ such that $A \cup L$ is obtained from $A \cup D$ by attaching handles of index $\leq n$. 
The geometric device in the proof of our lemma 2.1, using the family of domains $\left\{D_{t}\right\}_{t \in[0,1]}$ which increase from $D_{0}$ to $D_{1}=L$, accomplishes this by using only the classical Morse theory for manifolds without boundary.

\section{Proof of theorems 1.1 and 1.2}

Theorem 1.1 corresponds to the special case $K=\emptyset$ and $J=J_{X}$ of the following.

Theorem 3.1. Let $\left(X, J_{X}\right)$ be a Stein manifold with $\operatorname{dim}_{\mathbb{C}} X \neq 2$, let $K \subset X$ be a compact $\mathcal{H}\left(X, J_{X}\right)$-convex subset with smooth strongly $J_{X}$-pseudoconvex boundary, and let $A$ be a closed complex subvariety of $X$. Assume that $J$ is an almost complex structure on $X$ which is integrable in an open neighborhood of $A \cup K$, it agrees with $J_{X}$ in a neighborhood of $A$, and such that $K$ is a strongly $J$-pseudoconvex with $J$ Stein interior. Let $Y$ be a complex manifold with a distance function $d_{Y}$ induced by a Riemannian metric.

Given a continuous map $f: X \rightarrow Y$ which is J-holomorphic in a neighborhood of $K$ and such that $\left.f\right|_{A}: A \rightarrow Y$ is holomorphic, there exists for every $\epsilon>0$ a homotopy of pairs $\left(J_{t}, f_{t}\right)(t \in[0,1])$, where $J_{t}$ is an almost complex structure on $X$ and $f_{t}: X \rightarrow Y$ is a continuous map, satisfying the following:

(i) $J_{0}=J$, and $J_{t}$ agrees with $J$ in a neighborhood of $A \cup K$ for every $t \in[0,1]$,

(ii) the structure $J_{1}$ is integrable Stein on $X$ and $K$ is $\mathcal{H}\left(X, J_{1}\right)$-convex,

(iii) $f_{0}=f$, and $\left.f_{t}\right|_{A}=\left.f\right|_{A}$ for every $t \in[0,1]$,

(iv) for each $t \in[0,1]$ the map $f_{t}$ is J-holomorphic in a neighborhood of $K$ and satisfies $\sup _{x \in K} d_{Y}\left(f_{t}(x), f(x)\right)<\epsilon$, and

(v) the map $f_{1}: X \rightarrow Y$ is $J_{1}$-holomorphic.

If $J$ is integrable on $X$ then $J_{t}$ can be chosen integrable for every $t \in[0,1]$.

We emphasize that the almost complex structure $J$ need not be homotopic to $J_{X}$. In fact, the Stein structure $J_{X}$ is only used to obtain a correct handlebody decomposition of the pair $(X, A)$ (see remark 3.2 below).

Proof. Since $K$ is strongly $J_{X}$-pseudoconvex and $\mathcal{H}\left(X, J_{X}\right)$-convex, there exists a smooth strongly $J_{X}$-plurisubharmonic Morse exhaustion function $\rho: X \rightarrow \mathbb{R}$ such that $K=\{x \in X: \rho(x) \leq 0\}$ and $d \rho \neq 0$ on $b K=\{\rho=0\}$. Choose a sequence $c_{0}=0<c_{1}<c_{2} \ldots$ consisting of regular values of $\rho$, with $\lim _{j \rightarrow \infty} c_{j}=+\infty$. Let $K_{j}=\left\{x \in X: \rho(x) \leq c_{j}\right\}$. Set $f_{0}=f$ and $J_{0}=J$. Applying lemma 2.1 we can inductively construct sequences of maps $f_{j}: X \rightarrow Y$ and of almost complex structures $J_{j}$ satisfying the following for $j=1,2, \ldots$ :

(a) $J_{j}$ is integrable in a neighborhood of $A \cup K_{j}$ and it agrees with $J_{j-1}$ in a neighborhood of $A \cup K_{j-1}$,

(b) $K_{j}$ is strongly $J_{j}$-pseudoconvex with $J_{j}$-Stein interior, and $K_{j-1}$ is $J_{j}$-holomorphically convex in $K_{j}$,

(c) there is a homotopy of almost complex structures $J_{j, s}(s \in[0,1])$, with $J_{j, 0}=$ $J_{j-1}$ and $J_{j, 1}=J_{j}$, which is fixed in a neighborhood of $A \cup K_{j-1}$,

(d) the map $f_{j}: X \rightarrow Y$ is $J_{j}$-holomorphic in a neighborhood of $K_{j}$ and $\left.f_{j}\right|_{A}=$ $\left.f\right|_{A}$, and 
(e) there is a homotopy $f_{j, s}: X \rightarrow Y(s \in[0,1])$ which is fixed on $A$ such that $f_{j, 0}=f_{j-1}, f_{j, 1}=f_{j}$, and for every $s \in[0,1]$ the map $f_{j, s}$ is $J_{j-1}$-holomorphic in a neighborhood of $K_{j-1}$ and it satisfies

$$
\sup _{x \in K_{j-1}} d_{Y}\left(f_{j, s}(x), f_{j-1}(x)\right)<2^{-j-1} \epsilon .
$$

Indeed, assuming that we have already constructed the above sequences up to $j-1$, it suffices to apply lemma 2.1 with $K=K_{j-1}, L=K_{j}, f=f_{j-1}, J=J_{j-1}$, and $\epsilon$ replaced by $2^{-j-1} \epsilon$ to get the next complex structure $J_{j}$ and the next map $f_{j}$ satisfying the stated properties.

Condition (a) insures that the limit $\widetilde{J}=\lim _{j \rightarrow \infty} J_{j}$ exists and is an integrable complex structure on $X$ which agrees with $J$ in a neighborhood of $A \cup K$. The manifold $X$ is exhausted by the sequence of strongly $\widetilde{J}$-pseudoconvex domains $K_{j}$ with $\widetilde{J}$-Stein interior. Property (b) implies that $K_{j}$ is $\mathcal{H}(X, \widetilde{J})$-convex for $j=0,1,2, \ldots$ and hence the manifold $(X, \widetilde{J})$ is Stein. By combining the individual homotopies furnished by (c) we obtain a homotopy of almost complex structures on $X$ which connects $J$ to $\widetilde{J}$ and which is fixed in a neighborhood of $A \cup K$.

Properties (d) and (e) insure that the sequence of maps $f_{j}: X \rightarrow Y$ converges uniformly on compacts in $X$ to a $\widetilde{J}$-holomorphic map $\tilde{f}=\lim _{j \rightarrow \infty} f_{j}: X \rightarrow Y$ satisfying $\left.\widetilde{f}\right|_{A}=\left.f\right|_{A}$ and $\sup _{x \in K} d_{Y}(\widetilde{f}(x), f(x))<\epsilon$. Finally, condition (e) implies that the homotopies $f_{j, s}(s \in[0,1], j=1,2, \ldots)$ can be assembled into a homotopy from $f$ to $\tilde{f}$ which is fixed on $A$, holomorphic on $K$, and $\epsilon$-close to $f$ on $K$.

Changing the notation so that $\widetilde{J}$ is denoted $J_{1}$ and $\widetilde{f}$ is denoted $f_{1}$ we obtain the conclusion of theorem 3.1.

Remark 3.2. The Stein structure $J_{X}$ was used in the above proof only to insure that for every $j=1,2, \ldots$ there is a thickening $D_{j-1} \subset K_{j}$ of the set $K_{j-1} \cup\left(A \cap K_{j}\right)$ such that $A \cup K_{j}$ is obtained (up to a diffeomorphism) by attaching handles of index $\leq \operatorname{dim}_{\mathbb{C}} X$ to $A \cup D_{j-1}$. (In the proof of lemma 2.1 this was shown using the notation $K_{j}=L, K_{j-1}=K$ and $D_{j-1}=D_{0}$.) This leads to a proof of theorem 1.1 under the weaker conditions that $(X, J)$ is an almost complex manifold of real dimension $2 n \neq 4$ such that $J$ is integrable in a neighborhood of a closed Stein subvariety $A \subset X$, and $X$ is exhausted by an increasing sequence of compact strongly $J$-pseudoconvex domains $K_{0} \subset K_{1} \subset \ldots \subset \cup_{j=0}^{\infty} K_{j}=X$ such that every pair $\left(A \cup K_{j}, A \cup K_{j-1}\right)$ satisfies the above topological condition.

Proof of theorem 1.2. We shall use the same tools as in the proof of theorem 3.1, but will change the induction procedure. Unlike in theorem 3.1, the complex structure on $X$ will remain fixed during the entire proof.

Let $K_{0} \subset K_{1} \subset \cdots \subset \cup_{j=0}^{\infty} K_{j}=X$ be an exhaustion of $X$ by compact, smoothly bounded, strongly pseudoconvex sets as in the proof of theorem 3.1. Set $f_{0}=f$. We shall assume that $f_{0}$ is holomorphic in a neighborhood of $K_{0}$ (choosing $K_{0}=\emptyset$ if so desired.) Let $d_{Y}$ be a distance function on $Y$.

Given an $\epsilon>0$ we shall inductively construct a sequence of compact, smoothly bounded, strongly pseudoconvex sets $\emptyset=O_{-1} \subset O_{0} \subset O_{1} \subset \ldots \subset X$, a sequence of 
smooth diffeomorphisms $h_{j}: X \rightarrow X$, and a sequence of maps $f_{j}: X \rightarrow Y$ satisfying the following properties for $j=1,2, \ldots$.

(i) $h_{j}\left(K_{j}\right)=O_{j}$, and $h_{j}$ is diffeotopic to $h_{j-1}$ by a diffeotopy which is fixed in a neighborhood of $A \cup K_{j-1}$,

(ii) $O_{j-1}$ is holomorphically convex in $O_{j}$,

(iii) $f_{j}$ is holomorphic in an open neighborhood of $O_{j}$ and satisfies $\left.f_{j}\right|_{A}=\left.f\right|_{A}$,

(iv) there is a homotopy $f_{j, s}: X \rightarrow Y(s \in[0,1])$ such that $f_{j, 0}=f_{j-1}, f_{j, 1}=f_{j}$, the homotopy is fixed on $A$, each map $f_{j, s}(s \in[0,1])$ is holomorphic in a neighborhood of $O_{j-1}$, and

$$
\sup _{x \in O_{j-1}} d_{Y}\left(f_{j, s}(x), f_{j-1}(x)\right)<2^{-j-1} \epsilon, \quad s \in[0,1] .
$$

We begin by setting $O_{0}=K_{0}, h_{0}=i d_{X}$ and $f_{0, s}=f_{0}$ for all $s \in[0,1]$. Suppose inductively that we have already constructed our sequences up to an index $j \in \mathbb{Z}_{+}$; thus the map $f_{j}: X \rightarrow Y$ is holomorphic on $A$ and in an open neighborhood of $O_{j}$. Property (i) implies that $h_{j}$ equals the identity map in a neighborhood of $A \cup K_{0}$. Hence $O_{j} \cap A=K_{j} \cap A$, and this set is holomorphically convex in $A$ since $K_{j}$ is $\mathcal{H}(X)$-convex. The set $O_{j}$, being strongly pseudoconvex, admits a basis of open Stein (strongly pseudoconvex) neighborhoods in $X$. In this situation theorem 3.1 in [9] applies and furnishes a map $f_{j}^{\prime}: X \rightarrow Y$ which is holomorphic in an open neighborhood $V_{j} \supset A \cup O_{j}$ and which approximates $f_{j}$ as close as desired uniformly on $O_{j}$. Replacing $f_{j}$ by $f_{j}^{\prime}$ we may therefore assume that $f_{j}$ is holomorphic in an open set $V_{j} \supset A \cup O_{j}$.

Applying lemma 2.1 with $f=f_{j}, K=K_{j}$ and $L=K_{j+1}$ we find a compact domain $D_{j} \subset K_{j+1}$ with strongly pseudoconvex boundary (denoted $D_{0}$ in lemma 2.1) such that $\left(A \cap K_{j+1}\right) \cup K_{j} \subset D_{j}, K_{j+1}$ is obtained from $D_{j}$ by attaching finitely many handles of index $\leq n=\operatorname{dim}_{\mathbb{C}} X$, and $h_{j}\left(D_{j}\right) \subset V_{j}$. The last inclusion is trivially satisfied in a neighborhood of $A$ where $h_{j}$ coincides with the identity map, while outside this neighborhood $D_{j}$ can be chosen as close as desired to $K_{j}$; since $h_{j}\left(K_{j}\right)=O_{j} \subset V_{j}$, the inclusion follows.

Set $O_{j}^{\prime}=h_{j}\left(D_{j}\right)$. If the above approximations were chosen sufficiently close then $O_{j}^{\prime}$ is a compact set with smooth strongly pseudoconvex boundary (since $b O_{j}^{\prime}$ coincides with $b D_{j}$ near the subvariety $A$, and elsewhere $b O_{j}^{\prime}$ is $\mathcal{C}^{\infty}$-close to the strongly pseudoconvex hypersurface $\left.h_{j}\left(b K_{j}\right)=b O_{j}\right)$. Note that $O_{j}$ is holomorphically convex in $O_{j}^{\prime}$ provided that $D_{j}$ is chosen in a sufficiently small neighborhood of $\left(A \cap K_{j+1}\right) \cup K_{j}$. Applying the diffeomorphism $h_{j}$ to the above sets we see that $h_{j}\left(K_{j+1}\right)$ is diffeomorphic to a handlebody $O_{j+1}$ obtained from $O_{j}^{\prime}=h_{j}\left(D_{j}\right)$ by attaching finitely many handles of index $\leq n$.

We now proceed as in the proof of theorem 3.1. By Lemma 5.1 in [10] the above handles can be chosen such that the resulting handlebody $O_{j+1}$ has smooth strongly pseudoconvex boundary, $O_{j}^{\prime}$ is holomorphically convex in $O_{j+1}$, and there is a map $f_{j+1}: X \rightarrow Y$ which is holomorphic in a neighborhood of $O_{j+1}$, it agrees with $f_{j}$ on $A$, and $\sup _{x \in O_{j}} d_{Y}\left(f_{j+1}(x), f_{j}(x)\right)<2^{-j-2} \epsilon$. The same lemma provides a homotopy from $f_{j}$ to $f_{j+1}$ satisfying property (iv) for the index $j+1$.

Since $O_{j+1}$ is constructed from $O_{j}^{\prime}$ by using the topological data provided by the pair $D_{j} \subset K_{j+1}$ and since all handles used in the construction of $O_{j+1}$ are contained 
in $X \backslash A$, there exists a diffeomorphism $g_{j}: X \rightarrow X$ which maps $h_{j}\left(K_{j+1}\right)$ onto $O_{j+1}$ and which is diffeotopic to $i d_{X}$ by a diffeotopy which is fixed (equal the identity map) in a neighborhood of $A \cup O_{j}^{\prime}$. The map $h_{j+1}=g_{j} \circ h_{j}: X \rightarrow X$ is a diffeomorphism of $X$ which maps $K_{j+1}$ onto $O_{j+1}$ and is diffeotopic to $h_{j}$ by a diffeotopy which is fixed near $A \cup K_{j}$. The induction may now continue.

Properties (i)-(iv) insure that $\Omega=\cup_{j=0}^{\infty} O_{j} \subset X$ is a Stein domain which contains $A \cup K_{0}$, and the sequence $f_{j}$ converges uniformly on compacts in $\Omega$ to a holomorphic map $\widetilde{f}=\lim _{j \rightarrow \infty} f_{j}: \Omega \rightarrow Y$ satisfying $\left.\widetilde{f}\right|_{A}=\left.f\right|_{A}$ and $\sup _{x \in K_{0}} d_{Y}(\widetilde{f}(x), f(x))<\epsilon$. Also, there is a homotopy of maps $\Omega \rightarrow Y$ from $\left.f\right|_{\Omega}$ to $\widetilde{f}$ which is holomorphic on $K_{0}$ and is $\epsilon$-close to $f_{0}$ on $K_{0}$. Property (i) also gives a diffeomorphism $h=$ $\lim _{j \rightarrow \infty} h_{j}: X \rightarrow h(X)=\Omega$ which is diffeotopic to $i d_{X}$ and which equals the identity map in a neighborhood of $A$. It follows that the map $\tilde{f} \circ h: X \rightarrow Y$ is homotopic to $f$, thereby completing the proof of theorem 1.2.

\section{The case $\operatorname{dim}_{\mathbb{C}} X=2$}

The proof of lemma 2.1, and hence of theorems 1.1 and 1.2, breaks down when $X$ is a Stein surface $\left(\operatorname{dim}_{\mathbb{C}} X=2\right)$, the reason being that a certain framing obstruction may arise when trying to add a 2-handle with an embedded totally real core disc attached along a Legendrian knot to a given strongly pseudoconvex boundary in $X$. This obstruction in the proof has been pointed out by Eliashberg [3], and the Seiberg-Witten theory subsequently confirmed that it cannot be removed in general. In particular, there exist smooth, orientable, almost complex 4-manifolds $(X, J)$ with a handlebody decomposition without handles of index $>2$ which do not admit any Stein structure; one such example is the manifold $X=S^{2} \times \mathbb{R}^{2}=\mathbb{C P}^{1} \times \mathbb{C}$. (Many futher examples can be found in [13].) The key obstruction for the existence of a Stein structure is provided by the generalized adjunction inequality which states that for every closed, orientable, smoothly embedded 2-surface $S$ in a Stein manifold $X$, with the only exception of a null-homologous 2 -sphere, we have

$$
[S]^{2}+\left|c_{1}(X) \cdot S\right| \leq-\chi(S) .
$$

(See Chapter 11 in [15], or [24], for a proof, references to the original papers and further results.) Conversely, a closed embedded orientable 2-surface in an arbitrary complex manifold $X$ which satisfies the above inequality is isotopic to another embedding with a basis of tubular open Stein neighorhoods in $X$ [4].

On the other hand, Gompf proved that there always exist exotic Stein structures on any such 4-manifold $X[13,14]$. More precisely, given a smooth, almost complex 4-manifold $(X, J)$ with a Morse exhaustion function without critical points of Morse index $>2$, there exist a Stein surface $\left(X^{\prime}, J^{\prime}\right)$ and an orientation preserving homeomorphism $h: X \rightarrow X^{\prime}$ such that the class determined by the almost complex structure $J^{\prime}$ via $h$ agrees with the class of $J$.

Keeping the same hypotheses on $(X, J)$, the authors have shown in $[10, \S 7]$ that for any continuous map $f: X \rightarrow Y$ to a complex manifold $Y$, a Stein surface $\left(X^{\prime}, J^{\prime}\right)$ and a homeomorphism $h: X \rightarrow X^{\prime}$ in Gompf's theorem can be chosen such that there exists a $J^{\prime}$-holomorphic map $f^{\prime}: X^{\prime} \rightarrow Y$ with the property that the map $\widetilde{f}=f^{\prime} \circ h: X \rightarrow Y$ is homotopic to $f$. If in addition the almost complex structure $J$ 
on $X$ is integrable (but not necessarily Stein), one can realize such $\left(X^{\prime}, J^{\prime}\right)$ as an open $J$-Stein domain $\Omega \subset X$ which is homeomorphic to $X$ (theorem 1.2 in [10]; without considering mappings this is again due to Gompf [14]).

The constructions in $[13,14,10]$ use kinky discs and Casson handles at every place where a framing obstruction arises in the construction, together with the famous result of Freedman to the effect that a Casson handle is homeomorphic to a standard index two handle $\Delta_{2} \times \Delta_{2} \subset \mathbb{R}^{4}[11,12]$. By using the same tools, together with the methods explained in this paper, one can prove the following interpolation theorem which is the analogue of theorem 1.2 in the case $\operatorname{dim}_{\mathbb{C}} X=2$.

Theorem 4.1. Let $X$ be a Stein surface and let $A$ be a closed complex subvariety of $X$. Given a continuous map $f: X \rightarrow Y$ to a complex manifold $Y$ such that $\left.f\right|_{A}: A \rightarrow Y$ is holomorphic, there exist a Stein domain $\Omega \subset X$ containing $A$, a holomorphic map $\tilde{f}: \Omega \rightarrow Y$, and an orientation preserving homeomorphism $h: X \rightarrow \Omega$ which is homeotopic to $i d_{X}$ by a homeotopy that is fixed on a neighborhood $A$, such that the map $\tilde{f} \circ h: X \rightarrow Y$ is homotopic to $f$ relative to $A$.

This can be proved by modifying the proof of theorem 1.2 in $\S 3$ above, and the necessary modification is explained in the proof of theorem 1.2 in $[10, \S 7]$. To avoid excessive repetition we shall only recall the essential points.

Let $J$ denote the given Stein structure on $X$. We assume the notation used in the proof of theorem 1.2 in $\S 3$ above. In that proof it is explained how one obtains a strongly pseudoconvex handlebody $O_{j+1}$ by attaching handles of index $\leq n$ to a strongly pseudoconvex domain $O_{j}^{\prime}$. Each of the handles must have an embedded totally real core disc whose boundary circle is attached to the previous strongly pseudoconvex hypersurface along a Legendrian knot; this enables us to choose the next handlebody to be strongly pseudoconvex and to approximate the holomorphic map by a map which is holomorphic on a neighborhood of the new (larger) handlebody.

When $\operatorname{dim}_{\mathbb{C}} X=2$, a framing problem may arise for handles of index 2 , and a required totally real embedded core disc $M$ does not exist in general. As explained in [10] (and before that in [13]), the problem can be resolved by choosing an embedded core disc $M$ which is attached to the given strongly pseudoconvex domain $W \subset X$ along a Legendrian knot $b M \subset b W$, and then adding finitely many positive kinks to $M$. More precisely, we remove from $M$ finitely many small pairwise disjoint discs and glue along each of the resulting circles an immersed disc with one positive double point. (Fig. 4, borrowed from [10], shows a kink with a trivializing disc $\Delta$ which will be attached at the next step in order to cancel the superfluous loop at the double point $p$. A model kink used in [10] is provided by an explicit immersed Lagrangian sphere in $\mathbb{C}^{2}$, due to Weinstein [25].)

As explained in [10], kinking the core disc sufficiently many times gives an immersed disc which can be deformed to a totally real immersed disc $M^{\prime} \subset X \backslash \operatorname{IntW}$, attached to $b W$ along a Legendrian knot $b M^{\prime} \subset b W$. It is then possible to find a thin strongly pseudoconvex neighborhood $W^{\prime} \subset X$ of $W \cup M^{\prime}$ and a holomorphic map $W^{\prime} \rightarrow Y$ which approximates the given initial map $f: X \rightarrow Y$ uniformly on $W$. The manifold $W^{\prime}$ does not have the correct topology (it is not even homeomorphic to the domain obtained by attaching to $W$ a standard handle with an embedded core disc). The problem is partially corrected in the next stage of the construction by attaching to 


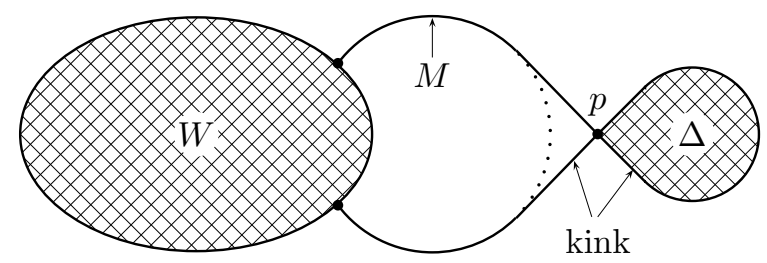

FIgURE 4. A kinky disc $M$ with a trivializing 2-cell $\Delta$

$W^{\prime}$ a trivializing 2-disc $\Delta$ at each of the kinky points in order to cancel the extra loop. Unfortunately the framing obstruction arises at this disc as well, requiring us to place another kink on $\Delta$ which will require a new trivializing disc, etc. The ensuing procedure is always infinite, it can be carried out in a small neighborhood of the initial kinky point in $M$, and it converges to an attached Casson handle which is homeomorphic to the standard 2-handle $\Delta_{2} \times \Delta_{2}$ (Freedman [11, 12]). Performing this construction inside $X$ gives a Stein domain $\Omega \subset X$ which is homeomorphic to $X$, but in general not diffeomorphic to $X$ due to the presence of Casson handles. A more precise description of this construction can be found in [10], and in [14] for the topological part. To insure that $\Omega$ contains the given subvariety $A \subset X$ we follow the proof of theorem 1.2 with these modifications.

\section{References}

1. ANDREOTTI, A., FRAENKEL, T., The Lefshetz theorem on hyperplane sections. Ann. Math., 69 (1959), 713-717.

2. DEMAILLY, J.-P., Cohomology of $q$-convex spaces in top degrees. Math. Z., 204 (1990), 283-295.

3. ELIASHBERG, Y., Topological characterization of Stein manifolds of dimension $>2$. Internat. J. Math., 1 (1990), 29-46.

4. FORSTNERIČ, F., Stein domains in complex surfaces. J. Geom. Anal., 13 (2003), 77-94.

5. - Noncritical holomorphic functions on Stein manifolds. Acta Math., 191 (2003), 143189.

6. - Holomorphic submersions from Stein manifolds. Ann. Inst. Fourier, 54 (2004), 19131942.

7. - Runge approximation on convex sets implies Oka's property. Ann. Math., 163 (2006), 689-707.

8. - Holomorphic flexibility properties of complex manifolds. Amer. J. Math., 128 (2006), 239-270.

9. — Extending holomorphic mappings from subvarieties in Stein manifolds. Ann. Inst. Fourier, 55 (2005), 733-751.

10. FORSTNERIČ, F., SLAPAR, M., Stein structures and holomorphic mappings. Math. Z. (2007). DOI: $10.1007 / \mathrm{s} 00209-006-0093-0$

11. FREEDMAN, M., The topology of four-dimensional manifolds. J. Diff. Geom., 17 (1982), 357453.

12. FREEDMAN, M. H., QUINN, F., Topology of 4-manifolds. Princeton Mathematical Series, 39. Princeton Univ. Press, Princeton, NJ, 1990.

13. GOMPF, R. E., Handlebody construction of Stein surfaces. Ann. Math., (2) 148 (1998), 619-693.

14. - Stein surfaces as open subsets of $\mathbb{C}^{2}$. J. Symplectic Geom. 3 (2005), 565-587. 
15. GOMPF, R. E., STIPSICZ, A. I., 4-manifolds and Kirby calculus. Graduate Studies in Math., 20. Amer. Math. Soc., Providence, RI, 1999.

16. GUNNING, R. C., ROSSI, H., Analytic functions of several complex variables. Prentice-Hall, Englewood Cliffs, 1965.

17. HAMM, H., Zum Homotopietyp Steinscher Räume. J. reine ang. Math., 338 (1983), 121-135.

18. —Zum Homotopietyp q-vollständiger Räume. J. reine ang. Math., 364 (1986), 1-9.

19. HÖRMANDER, L., An Introduction to Complex Analysis in Several Variables. Third ed. North Holland, Amsterdam, 1990.

20. LÁRUSSON, F., Mapping cylinders and the Oka principle. Indiana Univ. Math. J., 54 (2005), $1145-1159$

21. LEFSHETZ, S., L'analysis Situs et la Géometrie Algébrique. Paris, 1924.

22. MILnOR, J., Morse Theory. Ann. of Math. Studies, 51. Princeton Univ. Press, Princeton, 1963.

23. NEWLANDER, A., NIRENBERG, L., Complex analytic coordinates in almost complex manifolds. Ann. Math., (2) 65 (1957), 391-404.

24. OZBAGCI, B., STIPSICZ, A. I., Surgery on Contact 3-Manifolds and Stein Surfaces. SpringerVerlag, New York, 2005.

25. WeINSTEIN, A., Lectures on Symplectic Manifolds. Reg. Conf. Ser. Math., 29, Amer. Math. Soc., Providence, 1977.

Institute of Mathematics, Physics and Mechanics, University of Ljubluana, Jadranska 19, 1000 Ljubljana, Slovenia

E-mail address: franc.forstneric@fmf.uni-lj.si, marko.slapar@fmf.uni-lj.si 